\title{
PROCESO DE PRODUCCIÓN DE COLCHONES de espuma viscoelástica en el Perú
}

Caterina Strobbe*

Universidad de Lima, Perú

Recibido: 2 de octubre del 2019 / Aprobado: 14 de febrero del 2020

doi: $10.26439 /$ ing.ind2020.n038.4821

RESUMEN: El objetivo del artículo es exponer la viabilidad tecnológica de la instalación de una planta para colchones de espuma viscoelástica, con la finalidad de brindar mejor calidad de vida al usuario. Los temas más relevantes para el alcance del estudio son la definición del producto y la determinación del proceso de producción. Estas herramientas permiten entender el proceso, estableciendo los puntos clave para la elaboración de los colchones.

Palabras clave: espuma viscoelástica / colchones / polimerización / estudios de viabilidad / industria del colchón

\section{PRODUCTION PROCESS FOR MEMORY FOAM MATTRESSES IN PERU}

ABSTRACT: This article aims to define the technological viability of the installation of a memory foam mattress plant to provide users a better quality of life. The most relevant topics of the study are product definition and production process. These tools are important to understand the process and establish the critical points for mattress production.

Keywords: Memory foam / mattresses / polymerization / feasibility studies / mattress industry 


\section{INTRODUCCIÓN}

\subsection{Introducción a la industria de los colchones}

La industria de colchones se puede separar en dos grupos principales de acuerdo con la composición del producto: con resortes (muelles) y sin resortes. Dentro de los que tienen resortes hay distintas variedades; según el relleno, la forma y cantidad de resortes, y el número y grosor de las capas de soporte de la parte superior, ya sea una capa de algodón y tela, espuma de poliuretano, látex o viscoelástica. Dentro de los colchones sin resortes están los de espuma de poliuretano de baja densidad, espuma de látex y espuma viscoelástica (Viscoform, 2020).

En la actualidad, el material viscoelástico es el mejor en cuanto términos médicos y ha sido aprobado por la NASA en donde se iniciaron investigaciones a finales de los años sesenta para encontrar el material óptimo que resista altas presiones (Sonlab, 16 de noviembre del 2017). Este material se empezó a utilizar en colchones a inicios de los años noventa, por empresas de alto prestigio a nivel global, como es el caso de éxito de la empresa Tempur. Los colchones de espuma viscoelástica tienen la característica especial que evita que se deformen de manera permanente, teniendo una vida útil de 10 años (Anderson, 8 de febrero del 2019).

Por otro lado, el $90 \%$ del mercado de colchones son de muelles, estos colchones tienen una vida útil de 10 años, pero el colchón tiene que ir rotándose ya que va tomando la forma del usuario y se va deformando de manera permanente (Cantzler, 25 de abril del 2013). Además de deformarse, los resortes pueden llegar a romperse antes de su vida útil, creando incomodidades en el usuario. Como productos substitutos al colchón se encuentran los mats y los sofás cama, sin embargo, ninguno brinda las características de un colchón por tamaño, forma, calidad del material y comodidad de descanso.

\subsection{Problemática en el Perú}

La investigación de los colchones de espuma viscoelástica es un tema relevante, ya que el dolor de espalda y las lesiones a causa de ello son de alta importancia tanto en el Perú como en el mundo. Según el estudio realizado por Gardinalli Quiropraxia (centro especializado en el diagnóstico, tratamiento y prevención de los trastornos del sistema neuromusculoesquelético), se encontró que el 39,68 \% de los peruanos sufren de dolor cervical, el 14,29 \% de dolor dorsal y el 34,92 \% de dolor lumbar. Este número es elevado a comparación de otros síntomas comunes e importantes como dolores de cabeza, colesterol alto, presión alta y artritis, que solo representan el 4,76 \% (El $39 \%$ de peruanos sufre de dolores cervicales y eso incrementa el ausentismo laboral, 5 de febrero del 2016). 
Esto genera impacto para el país ya que ocasiona invalidez temporal. EsSalud informó que el $80 \%$ de los descansos médicos que generan se debe a dolores derivados de la columna (PYMEX, 16 de agosto del 2018).

\subsection{Impacto positivo de los colchones de espuma viscoelástica}

Al estar sentados el cuerpo humano genera mayor presión en la columna, por lo que es vital reposar con el cuerpo totalmente horizontal para que la gravedad y el peso de este no afecte la columna al ejercer más peso, como se puede apreciar en la figura 1.

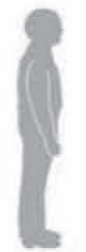

$100 \%$

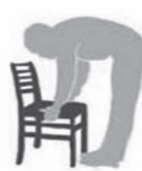

$200 \%$

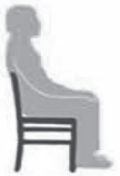

$150 \%$

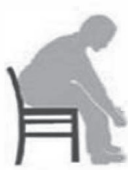

$250 \%$

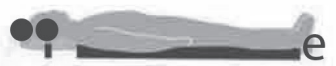

$25 \%$

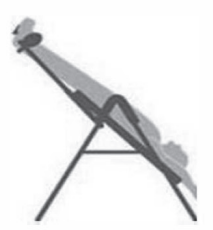

$0 \%$

Figura 1. Presión ejercida en la columna vertebral según posición

Fuente: Andrés (9 de mayo del 2019)

Una persona debe dormir en promedio 8 horas diarias, siendo momento vital para el descanso y relajación muscular. Por el exceso de carga laboral, esas horas de sueño recomendadas se ven reducidas; por lo tanto, se tienen que aprovechar al máximo para descansar todos los músculos y reparar el cuerpo (¿Cuánto tiempo es recomendable dormir según nuestra edad?, 3 de agosto del 2018).

Los colchones de espuma viscoelástica ofrecen una superficie única para cada usuario al ajustarse al cuerpo de cada uno. Otra característica importante del producto es que es una pieza entera de espuma para evitar el uso de resortes, como un colchón de muelles tradicional. Al no tener resortes y por la propiedad de la espuma de adaptarse al cuerpo, evita el rebote. Esto es significativo, ya que cuando la cama rebota se producen vibraciones que generan presión no deseada en el cuerpo. Estas características permiten que la columna descanse, reducen la presión en la columna, tanto la ejercida por la gravedad como la del rebote de los resortes, y permite que los músculos se relajen al no tener que cargar con todo el peso del cuerpo. 


\subsection{Método de investigación}

La investigación de la viabilidad tecnológica de la implementación de una planta de producción de colchones de espuma viscoelástica en el Perú se hará llevando a cabo los siguientes pasos:

- Definir el producto

- Definir el proceso de producción

- Definir el impacto del proceso: impacto ambiental e impacto en la salud y seguridad

- Realizar el balance de materia y definir la capacidad de la planta

- Elaborar el plano de la planta de producción

\section{DEFINICIÓN DEL PRODUCTO}

\subsection{Colchones de espuma viscoelástica}

El colchón de espuma viscoelástica tiene como característica principal adaptarse a la postura del usuario, regresando a su posición original sin deformación permanente. La espuma viscoelástica tiene como característica que se ajusta al cuerpo, de manera suave en el área deseada y firme donde el cuerpo necesita soporte, manteniendo la alineación de la columna. Además, redistribuye la presión reduciendo las molestias generadas por el propio peso del cuerpo al dormir, porque el apoyo del cuerpo en el colchón es completo y no se centra en ciertos puntos de apoyo. Al no deformarse permanentemente, el usuario no tiene necesidad de estar rotando el colchón cada año, por lo tanto, tiene una vida útil de aproximadamente 10 años, si se conserva en las condiciones adecuadas (Tempur, 2020).

\subsection{Definición del producto y de la calidad}

\subsubsection{Definición del producto}

El producto básico es un colchón de cama para el descanso humano; el producto real, un colchón de espuma viscoelástica. La densidad de la espuma es $56 \mathrm{~kg} / \mathrm{m}^{3}$ (Kang, Lee y Kim, 2011). La composición química del material se encuentra en la tabla 1. 
Tabla 1

Proporción de insumos para espuma viscoelástica de densidad $56 \mathrm{~kg} / \mathrm{m}^{3}$

\begin{tabular}{lr}
\hline Insumo & pphp [1] \\
\hline Polipropilenglicol & 100,00 \\
Tolueno diisocianato & 52,81 \\
Agua destilada & 1,60 \\
Cloruro de metileno & 2,0 \\
Surfactante de silicona & 1,0 \\
Catalizador de amina & 0,3 \\
& 157,71 \\
\hline
\end{tabular}

Nota 1: pphp, partes por 100 partes de poliol Fuente: Kang, Lee y Kim (2011)

La espuma tiene que ser cortada según las dimensiones convencionales de las camas, como se muestra en la figura 1, para formar el colchón y que quepa sobre cualquier somier para que no sea un inconveniente para el consumidor. Además, para un mejor cuidado del colchón, se va a cubrir en un forro de tela, producto que se va a adquirir de terceros, el cual se puede sacar para poder lavarlo.

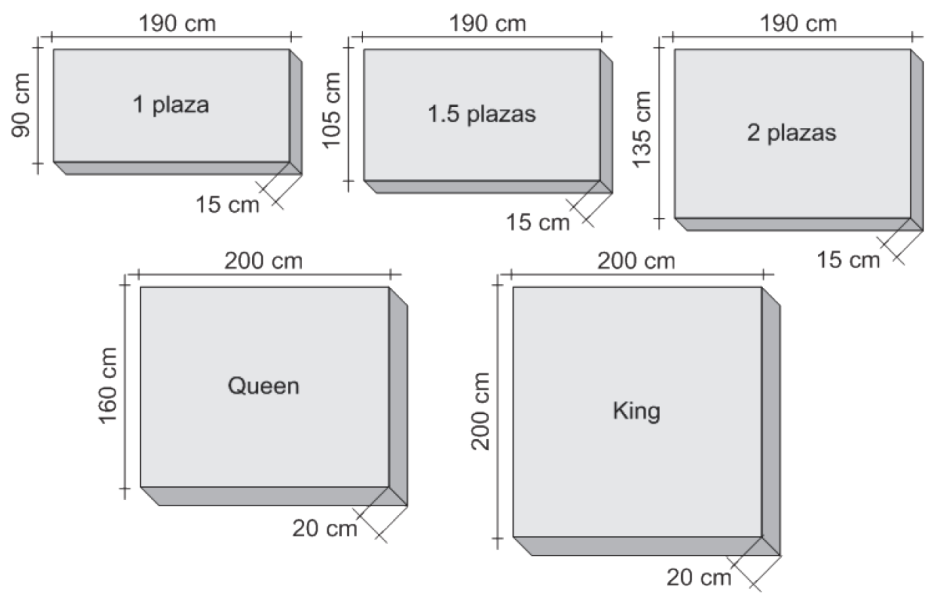

Figura 2. Diseño y medidas estándar de los colchones, según tamaño Elaboración propia 
Respecto al producto aumentado, se va a asesorar al cliente acerca del uso correcto del producto, explicándole los beneficios del material. Es importante explicarle al usuario cómo dormir y cuáles son las mejores posiciones, para que aproveche al máximo el producto que está adquiriendo.

\subsubsection{Calidad de los colchones de espuma viscoelástica}

El colchón de espuma viscoelástica tiene como característica principal adaptarse a la postura del usuario, regresando a su posición original sin deformación permanente. Este es un punto que distingue la calidad del producto; al no deformarse de manera permanente, el colchón tiene una vida útil de aproximadamente 10 años, si se conserva en las condiciones adecuadas (Tempur, 2020). Es posible obtener esta característica por la composición del producto y por el control en los puntos críticos durante el proceso.

La espuma puede tener diferentes densidades, dependiendo del tamaño de sus celdas, ya que una celda grande tiene mayor aire adentro, por lo tanto, es una espuma de menor densidad, y viceversa. La densidad del colchón es de $56 \mathrm{~kg} / \mathrm{m}^{3}$, esto permite que haya espacio entre las celdas, lo cual admite el flujo de aire sin que las celdas sean tan amplias que colapsen con el peso. Es importante que la densidad no se vea afectada durante el proceso de producción, ya que tiene un impacto directo en la calidad percibida por el usuario (Kang et al., 2011).

Otra característica destacable del producto es que es una pieza entera de espuma viscoelástica para evitar el uso de resortes, como un colchón de muelles tradicional, o evitar juntar varias espumas, y que esta esté hecha de materia prima no reciclada ya que altera la calidad del producto final. Además, es importante que el cliente esté seguro de lo que compra, por lo tanto, según la Resolución Minesterial n. 346-2013-PRODUCE (2013), deben declararse los materiales predominantes y si son materiales de primer uso o reciclados.

\subsection{Composición del producto}

A continuación, se presenta la composición de la espuma viscoelástica en la tabla 2 y en los párrafos que le siguen, la importancia de cada una de las materias primas utilizadas dentro de la fórmula. 
Tabla 2

Composición del producto

\begin{tabular}{lrcrr}
\hline Materia prima & \multicolumn{1}{c}{ pphp* } & Masa molar $(\mathrm{g} / \mathrm{mol})$ & \multicolumn{1}{c}{ Masa } & Proporción \\
\hline Polipropilenglicol & 100,00 & 76,10 & 7610,00 & $43,74 \%$ \\
Tolueno diisocianato & 52,81 & 174,20 & 9199,50 & $52,88 \%$ \\
Agua destilada & 1,60 & 18,00 & 28,80 & $0,17 \%$ \\
Cloruro de metileno & 2,00 & 84,93 & 169,86 & $0,98 \%$ \\
Surfactante de silicona & 1,00 & 357,36 & 357,36 & $2,05 \%$ \\
Catalizador de amina & 0,30 & 105,14 & 31,54 & $0,18 \%$ \\
& 157,71 & & 17397,06 & $100,00 \%$ \\
\hline
\end{tabular}

* Nota: pphp, partes por 100 partes de poliol

Fuente: Kang, Lee y Kim (2011)

- Polipropilenglicol: es un plastificante cuya función principal es descomponer las cadenas de polímeros para evitar la formación de cadenas largas que convierten a la estructura en una estructura frágil. La idea del plastificante es que le dé la suavidad y maleabilidad a la espuma (Gama, Ferreria y Barros-Timmons, 2018).

- Tolueno diisocianato: tiene como función principal reaccionar con el polipropilenglicol para formar la espuma. Esta amina le da resistencia a las paredes de las células que se forman. Es importante que sean resistentes para que no colapsen con el peso del usuario del colchón, perdiendo su característica de deformarse temporalmente. La resistencia se logra agregando el insumo químico en exceso, para que se forme urea, de tal manera que se crean puentes de hidrógeno dándole mayor rigidez a la espuma (Gama et al., 2018).

- Agua: importante insumo para que ocurra la reacción y se forme dióxido de carbono. Debe contar con la medida exacta de agua, ya que, a mayor cantidad, mayor $\mathrm{CO}^{2}$ se forma, obteniendo una espuma de menor densidad. De modo contrario, a menor cantidad de agua, resulta una espuma de mayor densidad. Por lo tanto, de este insumo depende la capacidad de soportar carga, puesto que está vinculado a la densidad de la espuma obtenida (Strakowska, Czlonka y Strzelec, 2019).

- Cloruro de metileno: es un agente soplante cuya función es dar suavidad a la espuma y a la vez controlar la densidad de la misma.

- Surfactante de silicona: este agente químico le da estabilidad a la espuma para que sea un producto inerte. Además, baja la tensión superficial para facilitar la reacción del tolueno diisocianato con el agua. Por último, les da resistencia a las celdas formadas durante la reacción para evitar el colapso de la espuma durante el proceso de polimerización. 


\section{PROCESO PRODUCTIVO}

\subsection{Proceso de producción}

El proceso inicia midiendo la cantidad necesaria de cada materia prima, siguiendo las proporciones de la tabla 2. Se vierte en el reactor con agitador el polipropilenglicol, el tolueno diisocianato, el agua destilada, el cloruro de metileno, el surfactante de silicona y el catalizador de amina, en las medidas respectivas. La mezcla se agita por 30 segundos a 1500 rpm para obtener una mezcla homogénea y que la reacción de polimerización aditiva se lleve a cabo (Strakowska et al., 2019). El proceso de polimerización aditiva es la reacción de monómeros, que al reaccionar forman cadenas más largas, brindando propiedades físicas, como elasticidad y fuerza, al producto final (Rogers, 2020). Durante esta etapa del proceso es necesario que los operarios utilicen equipos de protección personal, debido a que los gases emitidos por la reacción pueden irritar las vías respiratorias.

Una vez que la mezcla es homogénea, se debe vaciar en el molde, previamente, empapelado con papel sulfurado para impermeabilizar el molde y evitar que el pan (término utilizado para representar el molde principal de espuma viscoelástica que se produce durante la expansión máxima) se adhiera a las paredes. El vertido de la mezcla debe ser de forma rápida y continua porque la espuma va expandiéndose rápidamente y debe manejarse con cuidado el vaciado porque la reacción es exotérmica.

El pan obtenido se deja reposar por 40 minutos a temperatura ambiente, para que baje la alta temperatura que alcanzó por la reacción. Luego, se corta a las medidas establecidas según tamaño y lote, para quitar toda irregularidad, y se deja curar por 24 horas a temperatura ambiente, máximo $25^{\circ} \mathrm{C}$, en un lugar seco de máximo $70 \%$ de humedad (Kang et al., 2011).

Una vez curadas las piezas, se cortan a la medida del colchón que se desea fabricar, según las dimensiones de la figura 2. Se utiliza una sierra con cinta horizontal y una vertical para realizar los cortes de manera pareja, disminuyendo los errores e imprecisión del trabajo manual.

El colchón es forrado por una funda de algodón y poliéster para obtener el producto terminado. Esa funda se compra a un proveedor, hecha a la medida, e incluye un cierre para facilitar la operación. Finalmente, el colchón forrado es embalado en plástico para evitar cualquier tipo de contaminación. Todos estos trabajos se realizan sobre una mesa de trabajo cumpliendo las medidas ergonómicas para esta labor.

A continuación, se presenta el diagrama de flujo del proceso y el balance de materiales. 

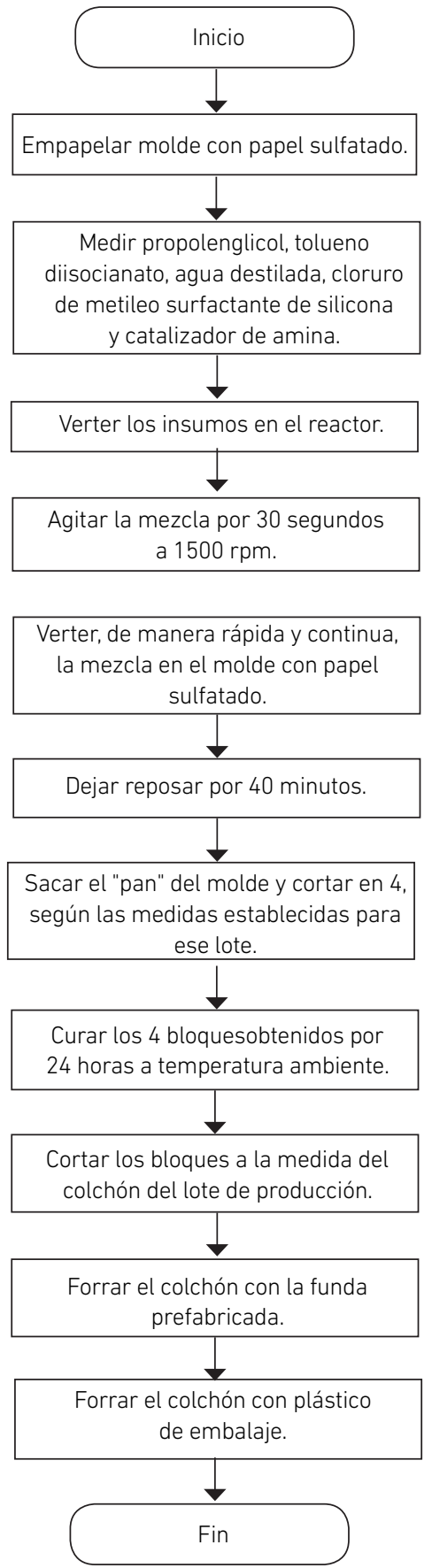

Figura 3. Diagrama de flujo del proceso productivo

Elaboración propia 


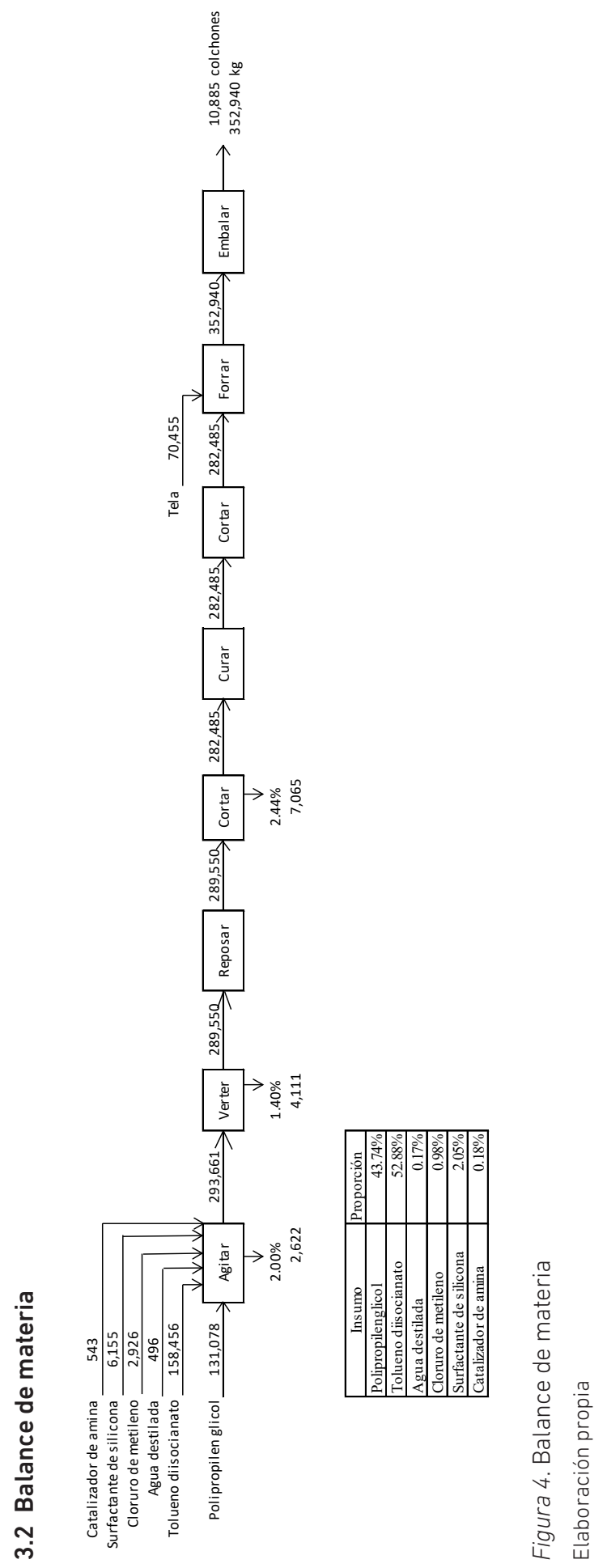




\subsection{Capacidad instalada}

\subsubsection{Selección de la maquinaria y equipos}

- Reactor: máquina donde sucede la reacción de polimerización al incorporar la materia prima. Tiene un agitador con angular mínima de 1500 rpm y es de fácil maniobra para su posterior vaciado, de manera rápida. La capacidad es de $200 \mathrm{~L}$, y la potencia de 2 a 10 HP.

- Cortadora horizontal y vertical: se adapta para distintos tamaños de colchones debido a que las dimensiones varían entre los lotes. Tiene una velocidad de corte $5 \mathrm{~m} / \mathrm{min}$, potencia de $6 \mathrm{~kW}$ y el modelo es double knife para corte vertical y horizontal.

- Balanza electrónica: tener una para mayor precisión al pesar los materiales. El rango de pesado es de 0 a $1500 \mathrm{~kg}$, con capacidad de lectura de $0,5 \mathrm{~kg}$, con opción a calibrarla.

- Moldes abiertos para cada tamaño de colchón: estos serán de madera, para darle soporte, forrados por el interior con silicona para que soporte altas temperaturas por la reacción exotérmica, y ayuda a que el pan no se adhiera a las paredes. Tendrán 4 ruedas en la parte inferior de modo que sea de fácil traslado, por el peso y tamaño, y, además, las 4 paredes se pueden abrir para así retirar la espuma una vez esté lista.

- Montacargas: importante para poder trasladar las parihuelas con el producto terminado y el producto en proceso, ya que son productos de gran volumen con peso significativo.

\subsubsection{Cálculo detallado del número de máquinas y operarios requeridos}

La planta trabaja 260 días al año, con 1 turno de 8 horas cada día, 5 días a la semana.

$$
H=260 \frac{\text { días }}{a \tilde{\text { ño }}} \times 1 \frac{\text { turno }}{\text { día }} \times 8 \frac{\text { horas }}{\text { turno }}=2080 \frac{\text { horas }}{a \tilde{n} o}
$$

Factor de eficiencia de 0,8 y 0,9 de utilización.

La fórmula para el cálculo de las máquinas es la siguiente:

número de máquinas $=\frac{\text { Cantidad a procesar }(P) \times \text { Tiempo estándar por máquina }(T)}{\text { Utilización }(U) \times \text { Eficiencia }(E) \times \text { Tiempo Disponible }(H)}$ 
A continuación, se detalla la cantidad de máquinas requeridas. Únicamente se utilizan máquinas en la reacción y en el corte, los procesos de verter, curar, forrar y embalar son manuales.

Tabla 3

Número de máquinas requeridas

\begin{tabular}{ccccccccc}
\hline Máquina & $\begin{array}{c}\text { Cantidad } \\
\text { a } \\
\text { procesar }\end{array}$ & $\begin{array}{c}\text { Unidad } \\
\text { utilizada }\end{array}$ & $\begin{array}{c}\text { Tiempo } \\
\text { estándar } \\
\text { (horas/ } \\
\text { unidad) }\end{array}$ & U & E & $\begin{array}{c}\text { Tiempo } \\
\text { disponible } \\
\text { (h) }\end{array}$ & $\begin{array}{c}\text { Cálculo del } \\
\text { número de } \\
\text { máquinas }\end{array}$ & $\begin{array}{c}\text { Número } \\
\text { de } \\
\text { máquinas }\end{array}$ \\
\hline Reactor & 10885 & unidad & 0,0729 & 0,9 & 0,8 & 2032 & 0,5535 & 1 \\
Cortadora & 10885 & unidad & 0,0429 & 0,9 & 0,8 & 2032 & 0,3268 & 1 \\
\hline
\end{tabular}

Elaboración propia

Al ser las máquinas semiautomáticas, se necesitarán: a) un operario para que prepare los insumos y ejecute la reacción, b) un operario en el área de corte y embalado y, c) además, otro encargado del almacén y movimiento de los productos, quien ayudará en la reacción a la hora que es transferida al molde. Por lo tanto, serán 3 los operarios.

\subsubsection{Cálculo de la capacidad instalada}

Luego de calcular el número de máquinas, se procedió a calcular la capacidad instalada, cuya fórmula es la siguiente:

Capacidad instalada $=\frac{\mathrm{kg}}{\text { hora }} \times \frac{\text { horas }}{\text { turno }} \times \frac{\text { turno }}{\text { día }} \times \frac{\text { días }}{a \text { ño }} \times$ factores $\times$ número de máquinas

En la tabla 4 se resalta que la capacidad instalada está dada por la cortadora, siendo esta máquina el cuello de botella, al ser 21530 toneladas la capacidad disponible en la planta. Esta capacidad representa 664005 colchones anuales. 
Proceso de producción de colchones de espuma viscoelástica en el Perú

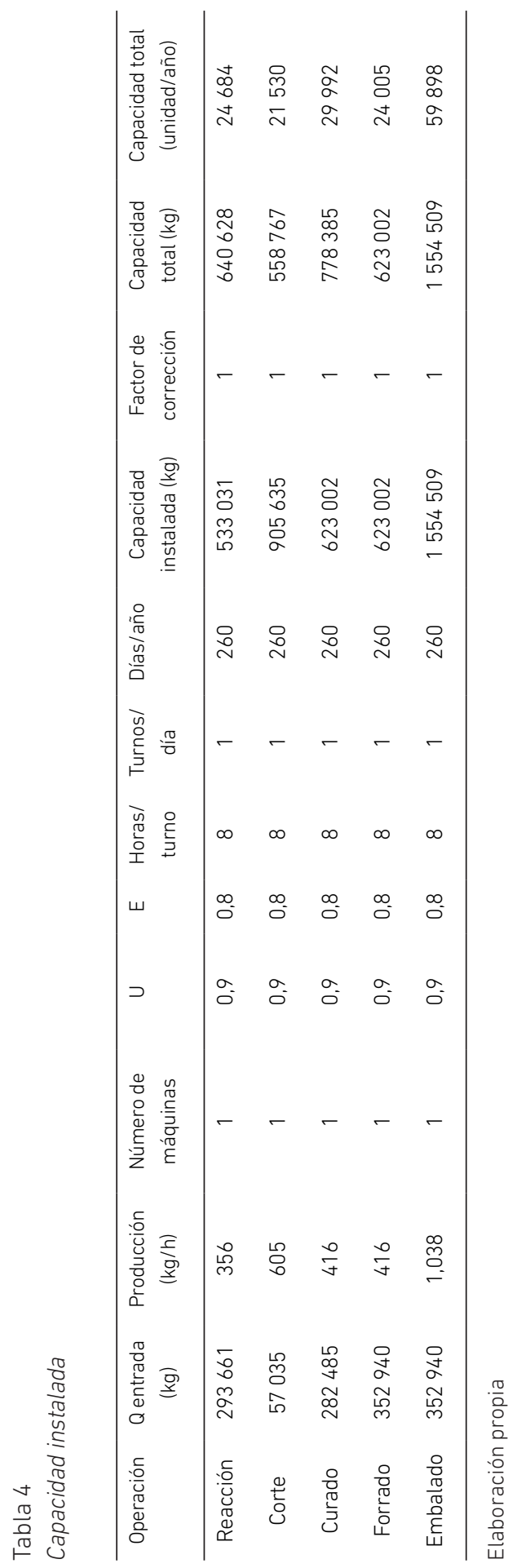




\section{SEGURIDAD Y SALUD OCUPACIONAL}

Deben cumplirse todas las normas de seguridad establecidas por el Estado peruano, según la Ley n. ${ }^{\circ} 29783$. En el capítulo III, artículo 26, la ley menciona que "garantizar la seguridad y salud en el trabajo es una responsabilidad conocida y aceptada en todos los niveles de la organización" (Ley de Seguridad y Salud en el Trabajo, 2011). Es importante que todos los empleados tengan presente el reglamento de seguridad y salud ocupacional, que se hagan capacitaciones constantes al personal, y que se supervise que se cumplan. A continuación, se detallan las medidas que se tomarán en consideración:

- Iluminación adecuada según área de trabajo: zona de producción y oficinas administrativas: 750 lux, almacén de materia prima 500 lux y almacén de producto terminado 300 lux.

- Tapones para personal en planta que está expuesto a más de 85 decibeles durante su jornada laboral de 8 horas, establecido por la Ley n. 29783.

- Uso de fajas lumbares para la carga de materiales pesados.

- Uso de mascarillas (respiradores) de seguridad con filtros de inhalación y válvula de exhalación para evitar la intoxicación con los gases de la reacción.

- Señalización de uso de EPP, según área, y ruta de evacuación.

- Contar con 6 extintores, 4 en la zona de producción y almacenes, debido a que el material es altamente inflamable, y 2 en las áreas administrativas.

La matriz IPER indica el nivel de riesgo de cada actividad realizada durante el proceso y las acciones a tomar para mitigarlos.

\section{Tabla 5}

Criterios para estimar la probabilidad del evento en la matriz IPER

\begin{tabular}{|c|c|c|c|c|}
\hline Índice & Personas expuestas & $\begin{array}{l}\text { Procedimientos } \\
\text { de trabajo }\end{array}$ & Capacitación & Severidad \\
\hline 1 & De 1 a 3 & $\begin{array}{l}\text { Existen, son } \\
\text { satisfactorios y } \\
\text { suficientes }\end{array}$ & $\begin{array}{l}\text { Personal entrenado, } \\
\text { conoce el peligro y lo } \\
\text { previene }\end{array}$ & $\begin{array}{l}\text { Lesión sin incapacidad. } \\
\text { Disconfort/Incomodidad }\end{array}$ \\
\hline 2 & De 4 a 12 & $\begin{array}{l}\text { Existen parcial- } \\
\text { mente y no son } \\
\text { satisfactorios ni } \\
\text { suficientes }\end{array}$ & $\begin{array}{l}\text { Personal parcial- } \\
\text { mente entrenado, } \\
\text { conoce el peligro } \\
\text { pero no toma accio- } \\
\text { nes de control }\end{array}$ & $\begin{array}{l}\text { Lesión con incapacidad } \\
\text { temporal, daño a la salud } \\
\text { reversible }\end{array}$ \\
\hline 3 & Más de 12 & No existen & $\begin{array}{l}\text { Personal no entre- } \\
\text { nado, no conoce el } \\
\text { peligro y no toma } \\
\text { acciones de control }\end{array}$ & $\begin{array}{l}\text { Lesión con incapacidad } \\
\text { permanente, daño a la } \\
\text { salud irreversible }\end{array}$ \\
\hline
\end{tabular}


Proceso de producción de colchones de espuma viscoelástica en el Perú

Tabla 6

Estimación de nivel de riesgos

\begin{tabular}{lll}
\hline Nivel de riesgo & Grado de riesgo & Criterio de significancia \\
\hline 5 & Trivial (T) & \\
5 a 8 & Tolerable (TO) & No significativo \\
9 a 16 & Moderado (MO) & \\
17 a 24 & Importante (IM) & Sí es significativo \\
25 a 36 & Intolerable (IT) & \\
\hline
\end{tabular}

Elaboración propia 


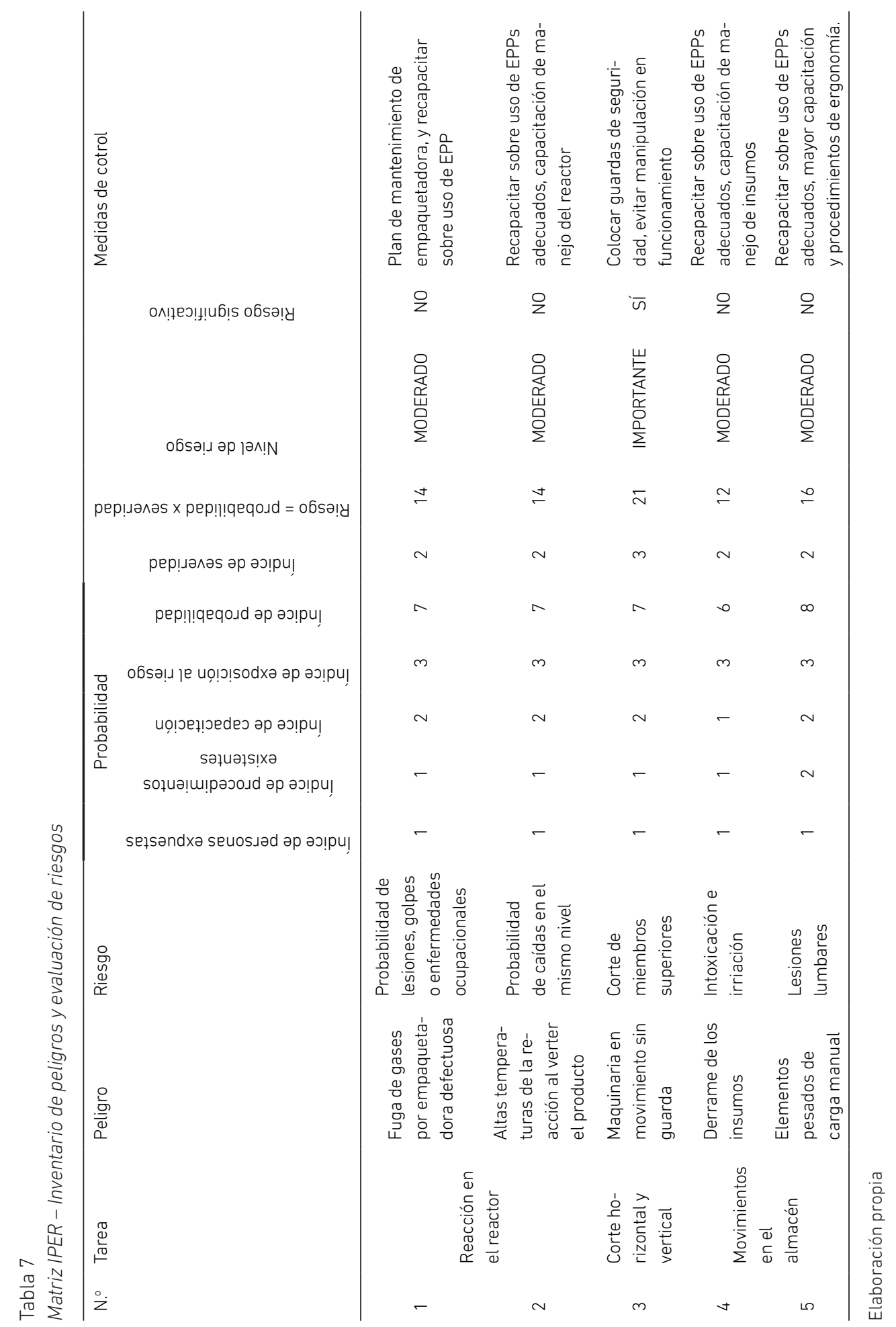




\section{CADENA DE VALOR}

La cadena de suministros se inicia con la compra de materia prima a proveedores locales. En el Perú hay varios proveedores que importan estos insumos químicos, por lo tanto, le facilita a la empresa la compra, al no tener que importarlo. Las ventajas de la compra local son: adquirir a un mejor precio, dado que el proveedor compra volúmenes mayores, reducir el gasto de almacenamiento y tener un flujo de caja positivo al ir comprando materia prima conforme se van dando las ventas.

El ideal es negociar con el proveedor local una compra cada 15 días, e ir considerando un stock adicional para 15 días más. Esto debe tenerse en cuenta, dado que el proveedor importa la materia prima, por lo tanto, es necesario contar con un tiempo de reacción si es que se quiebra el stock. Es importante contar con cotizaciones previas de los mismos químicos con otras empresas, para poder reaccionar rápido y no dejar de abastecer el mercado.

El segundo eslabón de la cadena es el almacenamiento de materias primas que, dependiendo del tipo de producto, se almacenará en racks o en tanques de almacenamiento de líquidos, puestos sobre los racks. Se utilizará el método $A B C$ para optimizar la distribución del almacén y el método PEPS (primero en entrar, primero en salir) para la entrada y salida de la materia prima y producto terminado.

El proceso de producción incluye el acarreo de materiales, manipulación y almacenamiento del producto en proceso. Se utilizan moldes con ruedas para facilitar el transporte de los lotes de espuma que se van a curar. El almacenamiento se realiza en parihuelas especiales que tienen las medidas de los colchones, según modelo, y son almacenados verticalmente.

Por último, la logística de salida incluye el almacenamiento interno y la distribución a los almacenes de los clientes. La distribución se terceriza y, principalmente, se lleva en camiones. Para no dañar el producto, se mantendrá cierta holgura en el interior del transporte para que los colchones no estén comprimidos entre sí.

Inicialmente, la empresa manejará el programa de producción como make to order (MTO). Esta política se basa en mejorar la estructura de costos, reduciendo al máximo la inversión en almacenamiento de producto terminado. Se fundamenta por el tipo de producto, siendo diferenciado en el mercado de colchones porque ningún otro tipo puede dar los beneficios que éste brinda. Como se mencionó previamente, en el mercado peruano se necesita concientizar sobre los beneficios del memory foam, por ello la empresa va a invertir en campañas de marketing y en los mismos puntos de venta para brindar información sobre el producto.

Esta política refleja por qué se debe seguir una estrategia pull. Se puede esperar una demanda constante debido al tipo de producto que manejamos. No existe una marcada 
estacionalidad para la compra de colchones, excepto que se espera que aumente en la etapa introductoria, por ser un producto existente, pero poco conocido. Este aumento sería fácilmente manejado por la capacidad de planta y el relativo poco tiempo en el que se produce un lote de colchones.

\section{CONCLUSIONES Y RECOMENDACIONES}

- Es necesario controlar todos los parámetros; cantidad de insumos, tiempo y velocidad de mezcla, humedad y temperatura para obtener la densidad de $56 \mathrm{~kg} / \mathrm{m}^{3}$ del colchón, así como los cambios en la mezcla, ya que todos estos influyen directamente en el producto.

- Con la información de la tecnología, procesos y materia prima existente, se puede concluir que el proceso es viable tecnológicamente. El proceso de polimerización para la producción de la espuma es un proceso de pocas etapas, facilitando el seguimiento de las condiciones críticas. Además, por medio de importación se consigue la maquinaria necesaria para la implementación y la materia prima para el proceso productivo.

- Se recomienda diversificar el portafolio de productos, por ejemplo, lanzar una línea de almohadas, o maquilar, porque aún se tiene capacidad sin utilizar. De esta forma se distribuyen los costos fijos y se mejora la rentabilidad de la empresa al aprovechar los activos.

\section{REFERENCIAS}

Anderson, R. (8 de febrero del 2019). Tempur my perfect fit. The history of Tempur and memory foam matresses. Recuperado de https://myperfectfit.tempur.com/ uk/2019/02/08/history-of-tempur-memory-foam-mattresses-nasa/

Andrés, S. (9 de mayo del 2019). Fisiolution, tus clínicas de confianza. Sedentarismo durante el tiempo de ocio. Recuperado de https://fisiolution.com/noticias/sedenta rismo-durante-el-tiempo-de-ocio/

Cantzler, S. (25 de abril del 2013). Evolución en el mercado del descanso 2012 vs. 2011. Recuperado de http://america-retail.com/opinion/evolucion-en-el-mercado -del-descanso-2012-vs-2011

¿Cuánto tiempo es recomendable dormir según nuestra edad? (3 de agosto del 2018). Gestión. Recuperado de https://gestion.pe/tendencias/salud-recomendabledormir-edad-240499-noticia/

El $39 \%$ de peruanos sufre de dolores cervicales y eso incrementa el ausentismo laboral.

(5 de febrero del 2016). Gestión. Recuperado de https://gestion.pe/tendencias/ 
management-empleo/39-peruanos-sufre-dolores-cervicales-incrementaausentismo-laboral-110954

Euro Moulders. (2 de junio del 2016). Guidelines for establishment of a safety management system in a flexible polyurethane foam plant. Recuperado de https://www. europur.org/publications/item/36-safety

Gama, N., Ferreria, A. y Barros-Timmons, A. (2018). Polyurethane foams: past, present and future. Materials Review, 11(10), 1841. doi:10.3390/ma11101841

Kang, S., Lee, S. y Kim, B. (2011). Shape memory polyurethane foams. Express Polymer Letters, 6(1) 63-69. doi:10.3144/expresspolymlett.2012.7

Ley $N^{\circ} 29783$. Ley de Seguridad y Salud en el Trabajo. (20 de agosto del 2011). Recuperado de http://www.mintra.gob.pe/LGT/ley_seguridad_salud_trabajo.pdf

PYMEX. (16 de agosto del 2018). Lumbalgia, la causa más común de descanso médico laboral. Recuperado de https://pymex.com/liderazgo/productividad/lumbalgia -la-causa-mas-comun-de-descanso-medico-laboral/

Resolución Ministerial n. 3 346-2013-PRODUCE. , Reglamento Técnico sobre Etiquetado de Colchones. (4 de diciembre del 2013). Ministerio de la Producción. Recuperado de http://www2.produce.gob.pe/dispositivos/publicaciones/rm346-2013-produce. pdf

Rogers, K. (2020). Polymerization Chemical Reaction. Encyclopaedia Britannica. Recuperado de https://www.britannica.com/science/polymerization

Sonlab. (16 de noviembre del 2017). ¿Por qué es importante la protección viscoelástica? Recuperado de https://www.sonlab.es/blog/2017/11/16/proteccionviscoelastica/

Strakowska, A., Czlonka, S., y Strzelec, K. (2019). POSS Compounds as Modifiers for Rigid Polyurethane Foams (Composites). Polymers, 11(7), 1092. doi:10.3390/ polym11071092

Tempur. (2020). Material benefits. Recuperado de https://au.tempur.com/tempurmaterial.html

Viscoform. (2020). Como elegir colchón: viscoelástico, latex, muelles. Recuperado de https://viscoform.es/como-elegir-colchon-viscoelastico-latex-muelleespumacion/ 
